\title{
Prognostic significance of lymphangiogenesis in pharyngolaryngeal carcinoma patients
}

\author{
Darío Garcia-Carracedo', Juan Pablo Rodrigo ${ }^{1,2}$, Aurora Astudillo ${ }^{1,3}$, Carlos Suarez Nieto ${ }^{1,2}$, Maria Victoria Gonzalez ${ }^{\text {* }}$
}

\begin{abstract}
Background: Lymphatic vessel spread is considered a major route for head and neck squamous cell carcinoma metastasis. Formation of new lymphatic vessels could facilitate the process, raising the malignant potential of these tumours. Recent identification of lymphatic markers allows the study of the lymphangiogenesis phenomenon. We searched for molecular events involved in the lymphangiogenic process that could have prognostic value in laryngeal/pharyngeal carcinoma patients.

Methods: 104 paraffin-embedded pharyngeal/laryngeal tumour samples were studied. Immunohistochemical analysis of podoplanin and double immunofluorescence analysis of Ki-67 and D2-40 were performed. Lymph vessel density (inside the tumour mass, at its periphery or considered as a whole) and the presence of tumour emboli inside lymphatics were recorded. The proliferative state of endothelial lymphatic cells was evaluated.

Results: Lymphatic vessels were detected inside the tumour mass (75\%) and in the surrounding tissue (80\%); some of them in a proliferative state. Tumour emboli were detected in a high proportion of the cases (45\%). Lymphatic vessel density was higher in the pharyngeal cases $(p=0.0029)$, in greater size $(p=0.039)$, more advanced stage primary tumours $(p=0.006)$ and in carcinomas of patients with affected nodes $(p=0.019)$. The presence of tumour emboli and a high global vessel density were indicators of poor prognosis (recorded as death from tumour) in the laryngeal group ( $p=0.015$ and $p=0.027$, respectively), but notably not in the pharyngeal one. Interestingly, high global vessel density showed a negative prognostic value among pathologically staged NO laryngeal carcinomas $(p=0.03)$.

Conclusions: The lymphangiogenic process correlated with aggressive tumour features ( $\mathrm{pN}$ category, tumour size, tumour stage), but might play different roles in tumours arising from different anatomic sites.

Our results suggest that detection of tumour emboli and assessment of global vessel density using the D2-40 antibody, may be useful in the clinical practice, as predictors of reduced survival among pNO laryngeal carcinoma patients.
\end{abstract}

\section{Background}

Head and neck squamous cell carcinoma (HNSCC) remains a significant cause of morbidity and mortality, afflicting 500.000 new cases worldwide each year[1]. The single most adverse independent prognostic factor for patients with HNSCC is the involvement of regional lymph nodes, but its accuracy could be improved.

Invasion of cells into the surrounding tissue and the destruction of normal tissue architecture are two hallmarks of malignant tumours. Lymphatic vessels serve as

\footnotetext{
* Correspondence: gonzalezvictoria@uniovi.es

'Instituto Universitario de Oncología del Principado de Asturias (IUOPA),

Universidad de Oviedo, Asturias, Spain

Full list of author information is available at the end of the article
}

the primary conduit for malignant tumour cell to regional lymph nodes[2]. In recent years, increasing evidence support that lymphangiogenesis is involved in the process of lymphatic spread in HNSCC. However, whether cancer cells can metastasize by expansion and invasion of preexisting peritumoural lymphatics, or by the formation and invasion of new lymphatics within tumours remains an unsolved question due to the difficulty in distinguishing lymphatics from blood vessels[3-8]. Recently, several lymphatic endothelium markers were identified and the most reliable one among them is podoplanin, which is recognised by the monoclonal D240 antibody with a high specificity and sensitivity [9-11].
C Biomed Central 
Using the D2-40 antibody in this study, the presence and density of lymphatic vessels were determined and quantified in laryngeal/pharyngeal carcinomas, and their potential prognostic values were assessed.

\section{Methods}

Patients

Paraffin-embedded tissues from 104 patients with pharyngeal or laryngeal squamous cell carcinoma (Table 1) who underwent resection of their tumours at the Hospital Universitario Central de Asturias (HUCA) (2000 2006) were obtained from the Pathology Department. Having a balanced number of cases for every clinicopathologic category was one of the patient inclusion criteria. Prior to the start the study was evaluated for approval according to the institutional review board's guidelines on ethical procedures. None of the patients had received radio/chemotherapy prior to resection or were thought to have distant metastasis at the time of diagnosis. Mean follow-up periods are shown in Table 1. Endpoints examined were nodal involvement, disease specific and overall survival.

\section{Immunohistochemical detection of lymphatic vessels}

$4 \mu \mathrm{m}$ formalin-fixed paraffin-embedded sections were incubated overnight at $54-56^{\circ} \mathrm{C}$, deparaffinized in xylene and rehydrated through decreasing graded ethanol solutions. After endogenous peroxidase activity suppression (3\% hydrogen peroxide, $10 \mathrm{~min}$ ) and antigen retrieval (boiling in $10 \mathrm{mM}$ citrate buffer, $\mathrm{pH}$ 6.0), immunostaining was performed with D2-40 mouse monoclonal antibody against human podoplanin (M2A antigen, Covance, California, USA) (1:100 dilution, $4^{\circ} \mathrm{C}$, overnight in a humid chamber). Staining was done by using the DakoCytomation Envision Plus peroxidase mouse system. The stained protein was visualized using the DAB solution (Dako), and lightly counterstained with Mayershaematoxilyn.

To ascertain the specificity of the antibody immunoreactivity, a negative control was carried out by exclusion of the primary antibody. In this case, immunolabeling was completely abolished.

\section{Evaluation of staining}

Quantitative analysis of the intratumoural and peritumoural lymphatic vessel density was performed by two independent observers (DGC and MVG) in a blinded fashion. Three fields with the highest lymphatic vascular density were identified (Olympus BX-51 microscope; x100 magnification), and the vessels were counted ( $\times 400$ magnification) both within the tumour area (intratumoural lymphatic density; ILD) and within an area 500 $\mu \mathrm{m}$ from the tumour border (peritumoural lymphatic density; PLD). Lymphatic vessel density (LVD),
Table 1 Clinicopathologic features of the laryngeal/ pharyngeal squamous cell carcinoma patients and their primary tumours $(\mathrm{N}=104)$

\begin{tabular}{|c|c|}
\hline Clinicopathologic feature & $\mathrm{N}^{\circ}$ cases $(\%)$ \\
\hline Age & Mean 60 (33-86) \\
\hline \multicolumn{2}{|l|}{ Sex } \\
\hline Male & $98(94.2)$ \\
\hline Female & $6(5.8)$ \\
\hline \multicolumn{2}{|l|}{ Tumour site } \\
\hline Hypopharynx & $18(17.5)$ \\
\hline Oropharynx & $31(30.1)$ \\
\hline Glottis & $30(29.1)$ \\
\hline Supraglottis & $24(23.3)$ \\
\hline \multicolumn{2}{|l|}{ T category } \\
\hline 1 & $19(18.3)$ \\
\hline 2 & $20(19.2)$ \\
\hline 3 & $30(28.9)$ \\
\hline 4 & $35(33.6)$ \\
\hline \multicolumn{2}{|l|}{ N category } \\
\hline 0 & $47(45.2)$ \\
\hline 1 & $8(7.7)$ \\
\hline 2 & $41(39.4)$ \\
\hline 3 & $8(7.7)$ \\
\hline \multicolumn{2}{|l|}{ Degree of differentiation } \\
\hline Well & $48(49)$ \\
\hline Moderate & $32(32.6)$ \\
\hline Poor & $18(18.4)$ \\
\hline \multicolumn{2}{|l|}{ Tumour stage $\left(\mathrm{TNM}^{\mathrm{a}}\right)$} \\
\hline 1 & $18(18.18)$ \\
\hline$\|$ & $10(10.10)$ \\
\hline III & $14(14.14)$ \\
\hline IV & $57(57.58)$ \\
\hline
\end{tabular}

Status

Alive without tumour $\quad 49$ (47)

Alive with tumour 3 (3)

Death of tumour 34 (33)

Death other causes 18 (17)

Recurrence

No 66 (66)

Yes 34 (34)

Toxic habits

Cigarette smoking 103 (99)

Alcohol consumption 91 (88)

Mean follow up (months) (range)

Whole population $23(10-67)$

$\begin{array}{ll}\text { Larynx } & 26(9-67)\end{array}$

$\begin{array}{ll}\text { Pharynx } & 20(10-66)\end{array}$

aTNM staging system of the International Union Against Cancer $-6^{\text {th }}$ Edition [20].

regardless of the vessel location with respect to the tumour, was also considered. Lymphatic density was defined as the number of lymphatic vessels per $\mathrm{mm}^{2}$. 
We also evaluated invasion of the podoplanin-positive lymphatic vessels by cancer cells. Lymphatic invasion (LI) was considered to be present if at least one tumour cell cluster (a tumour embolus) was clearly visible inside a podoplanin-positive vessel. Cases were classified as LI positive or negative depending on the presence or absence of tumour emboli, respectively.

\section{Immunofluorescence study}

Ten representative cases were selected on the basis of the images of the lymphatic vessels obtained in D2-40 IHC. For double immunostaining, deparaffined and rehydrated sections were rinsed in $0.05 \mathrm{M}$ Tris-buffered saline (TBS; $\mathrm{pH} 7.5$ ) containing $0.1 \%$ bovine serum albumin and $0.2 \%$ Triton X-100. Endogenous peroxidase activity and nonspecific binding were blocked with $3 \% \mathrm{H}_{2} \mathrm{O}_{2}$ and $50 \%$ fetal calf serum (Sigma), respectively, and the sections were incubated overnight with the primary antibodies in a humid chamber at $4^{\circ} \mathrm{C}$ : mouse $\mathrm{D} 2-40$ (M2A antigen, Covance, California, USA) and rabbit anti-ki67 (Abcam ab833). After the incubation with the primary antibodies, sections were incubated for $1 \mathrm{hr}$ at room temperature with Texas red-labelled sheep anti-rabbit immunoglobulin $G(\operatorname{IgG})$ and fluorescein isothiocyanate-conjugated sheep anti-mouse IgG, both diluted at 1:100 (Amersham). Finally, tissue sections were photographed with a confocal-laser scanning microscope (Bio-Rad MR-600; Servicio de Proceso de Imágenes de la Universidad de Oviedo).

\section{Statistical analysis}

For statistical purposes, clinicopathological features were dichotomized as: pT category: 1-2, 3-4; pN category: N0, N1-3; TNM stage: I-II, III-IV. The molecular data distributed among the different clinical groups of tumours were tested for significance employing the $\chi^{2}$ test for categorical variables and $t$-Student/ANOVA or Mann Whitney/ Kruskal-Wallis tests for parametric and non parametric continuous variables, respectively. Survival curves (disease-specific and overall) were calculated using the Kaplan-Meier product limit estimate. Differences between survival times were analyzed by the log-rank method. Multivariate Cox proportional hazards models were used to examine the relative impact of either variables demonstrated to be statistically significant in univariate analysis or those variables likely to have an effect on the outcome. P-values $<0.05$ (based on two-tailed statistical analysis) were considered statistically significant. All the statistical analyses were performed with the statistical software SPSS 12.0 (SPSS, Inc., Chicago, IL).

\section{Results}

Results are reported following the REMARK guidelines (REporting recommendations for tumor MARKer prognostic studies)[12].

\section{Lymphatic density quantification}

Immunohistochemical detection of podoplanin with D240 antibody shows strong specific reactivity with lymphatic endothelial cells, while the endothelial cells of blood vessels show no signal (Figure 1A, E).

D2-40-positive lymphatic vessels could be identified between islands of neoplastic epithelium (Figure 1B, F) and at the tumour periphery (Figure 1C, G). Podoplanin expression was also detected in tumour cells at the tumour border (Figure 1B-C).

The percentages of the cases with positive lymphatic vessel stainings, locations of the positive standings (ILD, PLD or LVD), mean values, and score ranges in the 104 HNSCC, 54 laryngeal and 50 pharyngeal carcinoma samples are summarized in Tables 2, 3 and 4.

\section{Proliferating lymph vessels}

Double immunofluorescence assay was performed with D2-40 and the proliferation marker ki-67 in ten representative cases. Vessels were deemed positive for proliferation when they harboured at least one ki-67 positive cell (Figure 2A-L) and were observed inside the tumour mass or in its periphery.

\section{Lymphatic invasion (LI)}

Podoplanin-positive carcinoma cells could be visualized inside lymph vessels (irrespective of vessel location) in $43 \%$ of the cases (44/103, LI positive) (Figure 1D, H). Figure 2M$\mathrm{P}$ shows the proliferation state (ki-67) of a D2-40 positive tumour mass inside a lymphatic vessel (LI positive case).

\section{Correlations with clinicopathologic features}

Considering the whole series for analysis, higher mean ILD, PLD and LVD values were significantly associated with greater tumour size, lymph node metastasis, advanced tumour stage and pharyngeal tumour site (Table 2). Tables 3 and 4 summarize ILD, PLD and LVD mean values and LI distribution among clinicopathological features in laryngeal and pharyngeal carcinomas, respectively. Of note, while no association was found in the pharyngeal group, LVD correlated with greater tumour size and more advanced tumour stage within the laryngeal population ( $\mathrm{p}<0.05$, each).

The normal glottis is a particularly interesting site, since it is a lymph vessel free region. In our series, 13 out of the 29 glottic cases (45\%) showed lymphatics within the tumour mass, 3 of which were T1 tumours.

Importantly, the presence of tumour emboli (lymphatic invasion) correlated with anatomic site (28\% in laryngeal tumours $v s .58 \%$ in pharyngeal tumours; $\mathrm{p}=0.002$ ) and were more frequently observed in tumours of patients with affected lymph nodes $(\mathrm{p}=0.001)$, in greater size tumours $(\mathrm{p}=0.006)$ and in more advanced stage tumours $(\mathrm{p}=0.000)$. 


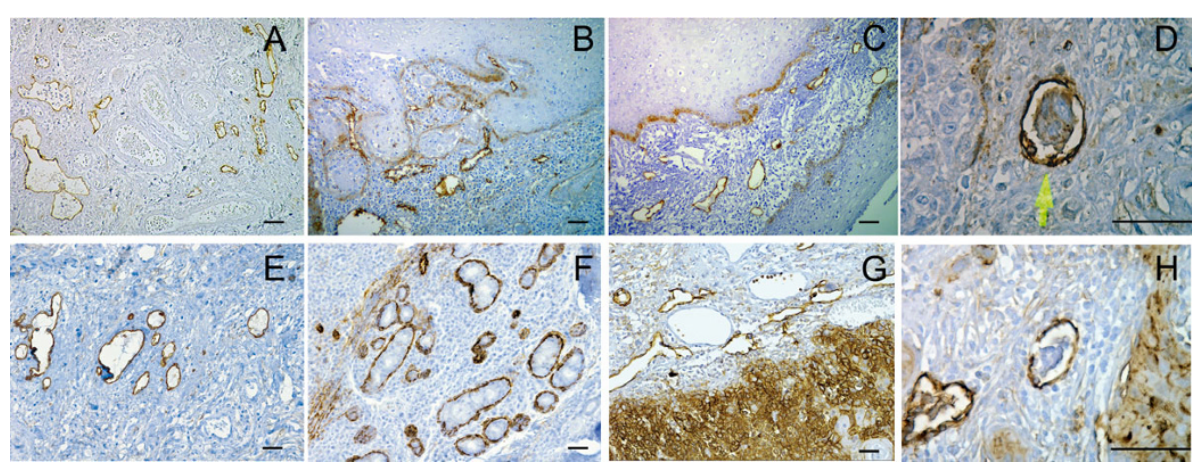

Figure 1 Immunohistochemical identification of lymphatic vessels. Immunohistochemical identification of lymphatic vessels using D2-40 antibody against podoplanin in an oropharyngeal (A) and a supraglottic tumour (E). Detection of intratumoural lymphatics in an oropharyngeal (B) and a supraglottic tumour (F). Detection of peritumoural lymphatics in a supraglottic tumour. The basal layer of the epithelium and the margin of the tumour mass gave positive signal for podoplanin (C). Detection of peritumoural lymphatics in a glottic tumour that stained positive for podoplanin (G). Example of one representative podoplanin positive tumour cell embolus inside a podoplanin positive lymphatic vessel of an oropharyngeal (D) and a supraglottic carcinoma (H). Scale bar, $250 \mu \mathrm{m}$.

Table 2 Correlations between lymphatic vessel density and clinicopathologic features in the HNSCC series (N = 104)

\begin{tabular}{|c|c|c|c|c|c|c|c|c|c|c|c|c|c|}
\hline \multirow{2}{*}{$\begin{array}{l}\text { ALL CASES } \\
\text { Frequency }\end{array}$} & \multicolumn{3}{|c|}{ ILD } & \multicolumn{3}{|c|}{ PLD } & \multicolumn{3}{|c|}{ LVD } & \multicolumn{4}{|c|}{ Lymphatic Invasion } \\
\hline & \multicolumn{3}{|c|}{$75 \%(78 / 104)$} & \multicolumn{3}{|c|}{$80 \%(83 / 104)$} & \multicolumn{3}{|c|}{$94 \%(98 / 104)$} & \multicolumn{4}{|c|}{$42.72 \%(44 / 103$} \\
\hline Mean (S.E.) & \multicolumn{3}{|c|}{$14.8(1.33)$} & \multicolumn{3}{|c|}{$22.0(1.59)$} & \multicolumn{3}{|c|}{$22.3(1.19)$} & & & & \\
\hline \multirow[t]{2}{*}{ Score range } & \multicolumn{3}{|c|}{ 0-78.4 } & \multicolumn{3}{|c|}{ 0-82.2 } & \multicolumn{3}{|c|}{ 0-79.3 } & & & & \\
\hline & $\mathbf{N}$ & Mean range & $p^{\#}$ & $\mathbf{N}$ & Mean (S.E) & $p^{*}$ & $\mathbf{N}$ & Mean (S.E) & $\mathrm{p}^{\#}$ & Absent & Present & Total & $\mathrm{p}^{*}$ \\
\hline \multicolumn{14}{|c|}{ Anatomic site } \\
\hline Pharynx & 50 & 63.34 & 0.0004 & 50 & $27.41(2.57)$ & 0.0072 & 50 & $26.47(1.92)$ & 0.0029 & 21 & 29 & 50 & 0.002 \\
\hline Larynx & 54 & 42.46 & & 54 & $18.47(2.03)$ & & 54 & $18.98(1.56)$ & & 38 & 15 & 53 & \\
\hline Total & 104 & & & 104 & & & 104 & & & 59 & 44 & 103 & \\
\hline \multicolumn{14}{|l|}{$\overline{\text { pT }}$} \\
\hline $\mathrm{T} 1-2$ & 39 & 39.09 & 0.0000 & 39 & $19.19(2.47)$ & 0.099 & 39 & $19.20(2.03)$ & 0.039 & 29 & 10 & 39 & 0.006 \\
\hline T3-4 & 65 & 60.55 & & 65 & $24.91(2.21)$ & & 65 & $24.61(1.60)$ & & 30 & 34 & 64 & \\
\hline Total & 104 & & & 104 & & & 104 & & & 59 & 44 & 103 & \\
\hline \multicolumn{14}{|c|}{ Nodal involvement } \\
\hline Free & 47 & 42.44 & 0.0020 & 47 & $18.60(2.37)$ & 0.0230 & 47 & $19.34(1.67)$ & 0.0190 & 35 & 12 & 47 & 0.001 \\
\hline Affected & 57 & 60.80 & & 57 & $26.20(2.27)$ & & 57 & $25.24(1.82)$ & & 24 & 32 & 56 & \\
\hline Total & 104 & & & 104 & & & 104 & & & 59 & 44 & 103 & \\
\hline \multicolumn{14}{|c|}{ Tumour Stage } \\
\hline$|-| \mid$ & 28 & 32.98 & 0.0001 & 28 & $14.67(2.44)$ & 0.0010 & 28 & $16.89(2.25)$ & 0.0060 & 24 & 4 & 28 & 0.0000 \\
\hline III-IV & 71 & 56.71 & & 71 & $25.83(2.14)$ & & 71 & $24.76(1.57)$ & & 31 & 38 & 69 & \\
\hline Total & 99 & & & 99 & & & 99 & & & 55 & 42 & 97 & \\
\hline \multicolumn{14}{|l|}{ Recurrence } \\
\hline No & 66 & 50.98 & 0.8167 & 66 & $20.29(2.17)$ & 0.0729 & 66 & $21.15(1.54)$ & 0.1649 & 38 & 27 & 65 & 0.8050 \\
\hline Yes & 34 & 49.57 & & 34 & $26.86(2.78)$ & & 34 & $25.04(2.47)$ & & 19 & 15 & 34 & \\
\hline Total & 100 & & & 100 & & & 100 & & & 57 & 42 & 99 & \\
\hline
\end{tabular}

ILD, Intratumoural Lymphatic Density; PLD, Peritumoural Lymphatic Density; LVD, Lymphatic Vessel Density

S.E., Standard Error

$\mathrm{p}^{\#}$ (U Mann-Whitney); $\mathrm{p}^{*}$ (t Student); $\mathrm{p}^{*}$ (chi square)

Frequencies (cases with lymph vessels), mean values and score ranges of ILD, PLD and LVD in the whole series (N=104). Comparison of mean ILD, PLD and LVD values among different categories of clinico-pathological features. Distribution of tumours according to lymphatic invasion (LI) and clinicopathological features. 
Table 3 Correlations between lymphatic vessel density and clinicopathologic features in the laryngeal carcinoma series $(\mathbf{N}=\mathbf{5 4})$

\begin{tabular}{|c|c|c|c|c|c|c|c|c|c|c|c|c|c|}
\hline \multirow{2}{*}{$\frac{\text { LARYNX }}{\text { Frequency }}$} & \multicolumn{3}{|c|}{ ILD } & \multicolumn{3}{|c|}{ PLD } & \multicolumn{3}{|c|}{ LVD) } & \multicolumn{4}{|c|}{ Lymphatic Invasion } \\
\hline & \multicolumn{3}{|c|}{$57.41 \%(31 / 54)$} & \multicolumn{3}{|c|}{$55.56 \%(30 / 54)$} & \multicolumn{3}{|c|}{$87.04 \%(47 / 54)$} & \multicolumn{4}{|c|}{$28.30 \%(15 / 53)$} \\
\hline Mean (S.E.) & & $10.59(1.65)$ & & & $18.47(2.03)$ & & & $18.98(1.56)$ & & & & & \\
\hline \multirow[t]{2}{*}{ Score range } & & $0-41.56$ & & & $0-60.45$ & & & $0-44.71$ & & & & & \\
\hline & $\mathbf{N}$ & Mean range & p\# & $\mathbf{N}$ & Mean (S.E) & $\mathrm{p} \neq$ & $\mathrm{N}$ & Mean (S.E) & $\mathrm{p} \neq$ & Absent & Present & Total & $\mathrm{p}^{*}$ \\
\hline \multicolumn{14}{|c|}{ Anatomic site } \\
\hline Glottis & 30 & 23.45 & 0.028 & 30 & $15.22(2.43)$ & 0.073 & 30 & $16.14(2.06)$ & 0.042 & 23 & 6 & 29 & 0.176 \\
\hline Supraglottis & 24 & 32.56 & & 24 & $22.54(3.30)$ & & 24 & $22.52(2.24)$ & & 15 & 9 & 24 & \\
\hline Total & 54 & & & 54 & & & 54 & & & 38 & 15 & 53 & \\
\hline \multicolumn{14}{|l|}{$\overline{\mathrm{pT}}$} \\
\hline T1-2 & 28 & 22.05 & 0.006 & 28 & $15.95(2.85)$ & 0.2 & 28 & $15.98(2.38)$ & 0.044 & 25 & 3 & 28 & 0.003 \\
\hline T3-4 & 26 & 33.37 & & 26 & $21.19(2.86)$ & & 26 & $22.20(1.84)$ & & 13 & 12 & 25 & \\
\hline Total & 54 & & & 54 & & & 54 & & & 38 & 15 & 53 & \\
\hline \multicolumn{14}{|c|}{ Nodal involvement } \\
\hline Free & 38 & 25.38 & 0.112 & 38 & $16.63(2.23)$ & 0.165 & 38 & $17.72(1.81)$ & 0.219 & 30 & 8 & 38 & 0.062 \\
\hline Affected & 16 & 32.53 & & 16 & $22.85(4.27)$ & & 16 & $21.96(3.03)$ & & 8 & 7 & 15 & \\
\hline Total & 54 & & & 54 & & & 54 & & & 38 & 15 & 53 & \\
\hline \multicolumn{14}{|c|}{ Tumour Stage } \\
\hline I-II & 24 & 20.92 & 0.004 & 24 & $13.95(2.52)$ & 0.045 & 24 & $15.05(2.35)$ & 0.023 & 20 & 4 & 24 & 0.073 \\
\hline III-IV & 30 & 32.77 & & 30 & $22.09(2.92)$ & & 30 & $22.12(1.94)$ & & 17 & 11 & 28 & \\
\hline Total & 54 & & & 54 & & & 54 & & & 37 & 15 & 52 & \\
\hline \multicolumn{14}{|l|}{ Recurrence } \\
\hline No & 43 & 28.35 & 0.169 & 43 & $17.02(2.28)$ & 0.218 & 43 & $18.60(1.80)$ & 0.685 & 29 & 13 & 42 & 0.492 \\
\hline Yes & 10 & 21.20 & & 10 & $23.55(4.7)$ & & 10 & $20.28(3.57)$ & & 8 & 2 & 10 & \\
\hline Total & 53 & & & 53 & & & 53 & & & 37 & 15 & 52 & \\
\hline
\end{tabular}

ILD, Intratumoural Lymphatic Density; PLD, Peritumoural Lymphatic Density; LVD, Lymphatic Vessel Density

S.E., Standard Error

$\mathrm{p}^{\#}$ (U Mann-Whitney); $\mathrm{p}^{*}$ (t Student); $\mathrm{p}^{*}$ (chi square)

Frequencies (cases with lymph vessels), mean values and score ranges of ILD, PLD and LVD in laryngeal carcinoma population (N=54). Comparison of mean ILD, PLD and LVD values among different categories of clinico-pathological features. Distribution of tumours according to lymphatic invasion (LI) and clinicopathological features.

\section{Survival analysis}

The five-year disease specific and overall survival rates for the whole population were $52 \%$ and $28 \%$, respectively. As expected, tumours arising in the larynx, those with T1-2 category, stage I-II and patients without lymph node involvement, had a better prognosis $(\mathrm{p}<0.05)$.

For the survival analysis, lymphatic vessel variables (ILD, PLD, LVD) were categorized into high vessel density cases $v s$. the rest, attending to the median value of the lymph vessel density in the positive population (Table 5). In this way, a high LVD (irrespective of vessel location) had a negative impact on disease specific survival $(p=0.045)$ and on overall survival $(p=0.0564)$. In addition, disease specific (Figure 3A) and overall survival were worse for patients with tumour emboli within the vessels (LI positive) ( $p=0.045$ and $p=0.0312$, respectively). Importantly, these results were observed for the laryngeal tumours (high LVD $(\mathrm{p}=0.027)$, presence of emboli; $\mathrm{p}=0.015$ ) (Figure 3B) but not in the pharyngeal ones.
A very interesting observation could be made for the 47 HNSCC patients with pN0 tumours (38 laryngeal and 9 pharyngeal): 19 cases showing a high LVD (14 laryngeal and 5 pharyngeal) had a poorer prognosis than the rest $(\mathrm{p}=0.005)$ (Figure $3 \mathrm{C})$. Among the group of $38 \mathrm{pNO}$ laryngeal tumours a high LVD was the only marker of poor prognosis in univariate analysis $(\mathrm{p}=0.03)$ (Figure $3 \mathrm{D})$.

None of these variables reached statistical significance when multivariate Cox analysis was performed.

\section{Discussion}

In the present study we investigated the existence of tumour lymphangiogenesis in a cohort of 104 laryngeal/ pharyngeal carcinoma patients and evaluated whether ILD, PLD or LVD correlated with the presence of lymph node metastasis; we also addressed the prognostic significance of the above parameters on the disease specific and overall survival of these patients.

As previously described, podoplanin was detected in tumour cells [13] and lymphatic vessels were observed 
Table 4 Correlations between lymphatic vessel density and clinicopathologic features in the laryngeal carcinoma series $(\mathbf{N}=\mathbf{5 0})$

\begin{tabular}{|c|c|c|c|c|c|c|c|c|c|c|c|c|c|}
\hline PHARYNX & & ILD & & & PLD & & & LVD & & & mphatic & nvasion & \\
\hline Frequency & & $90 \%(45 / 50$ & & & $90 \%(45 / 50)$ & & & $96 \%(48 / 50)$ & & & $58 \%(29$ & (50) & \\
\hline Mean (S.E.) & & $19.14(1.97)$ & & & $27.41(2.57)$ & & & $26.47(1.92)$ & & & & & \\
\hline Score range & & $0-78.39$ & & & $0-82.17$ & & & $8.5-79.34$ & & & & & \\
\hline & $\mathrm{N}$ & Mean (S.E) & $\mathrm{p} ¥$ & $\mathbf{N}$ & Mean (S.E) & $\mathrm{p} ¥$ & $\mathrm{~N}$ & Mean range & $\mathrm{p} \#$ & Absent & Present & Total & $\mathrm{p}^{*}$ \\
\hline Anatomic site & & & & & & & & & & & & & \\
\hline Hypopharynx & 18 & $19.92(3.48)$ & 0.8600 & 18 & 30.87 (3.91) & 0.3470 & 18 & 29.67 & 0.0810 & 12 & 6 & 18 & 0.0230 \\
\hline Oropharynx & 31 & $19.18(2.45)$ & & 31 & $25.70(3.46)$ & & 31 & 22.29 & & 9 & 22 & 31 & \\
\hline Total & 49 & & & 49 & & & 49 & & & 21 & 28 & 49 & \\
\hline pT & & & & & & & & & & & & & \\
\hline T1-2 & 11 & $14.71(3.53)$ & 0.236 & 11 & $27.45(3.12)$ & 0.993 & 11 & 29.18 & 0.343 & 4 & 7 & 11 & 0.668 \\
\hline T3-4 & 39 & $20.39(2.30)$ & & 39 & $27.39(3.11)$ & & 39 & 24.46 & & 17 & 22 & 39 & \\
\hline Total & 50 & & & 50 & & & 50 & & & 21 & 29 & 50 & \\
\hline Nodal involve & & & & & & & & & & & & & \\
\hline Free & 9 & $16.30(3.90)$ & 0.3330 & 9 & $26.94(7.77)$ & 0.3450 & 9 & 27.50 & 0.6550 & 5 & 4 & 9 & 0.3630 \\
\hline Affected & 41 & $19.77(2.25)$ & & 41 & $27.51(2.69)$ & & 41 & 25.06 & & 16 & 25 & 41 & \\
\hline Total & 50 & & & 50 & & & 50 & & & 21 & 29 & 50 & \\
\hline Tumour Stag & & & & & & & & & & & & & \\
\hline$|-| \mid$ & 4 & $12.12(7.01)$ & 0.3720 & 4 & $19.05(8.74)$ & 0.3650 & 4 & 28.13 & 0.4280 & 4 & 0 & 4 & 0.0100 \\
\hline III-IV & 41 & $19.60(2.29)$ & & 41 & $28.57(2.99)$ & & 41 & 22.50 & & 14 & 27 & 41 & \\
\hline Total & 45 & & & 45 & & & 45 & & & 18 & 27 & 45 & \\
\hline Recurrence & & & & & & & & & & & & & \\
\hline No & 23 & $19.19(2.37)$ & 0.9140 & 23 & $26.41(4.34)$ & 0.7410 & 23 & 24.39 & 0.8480 & 9 & 14 & 23 & 0.6420 \\
\hline Yes & 24 & $18.73(3.45)$ & & 24 & $28.24(3.43)$ & & 24 & 23.63 & & 11 & 13 & 24 & \\
\hline Total & 47 & & & 47 & & & 47 & & & 20 & 27 & 47 & \\
\hline
\end{tabular}

ILD, Intratumoural Lymphatic Density; PLD, Peritumoural Lymphatic Density; LVD, Lymphatic Vessel Density

S.E., Standard Error

$\mathrm{p}^{\#}$ (U Mann-Whitney); $\mathrm{p}^{*}$ (t Student); $\mathrm{p}^{*}$ (chi square)

Frequencies (cases with lymph vessels), mean values and score ranges of ILD, PLD and LVD in pharyngeal population (N=50). Comparison of mean ILD, PLD and LVD values among different categories of clinico-pathological features. Distribution of tumours according to lymphatic invasion (LI) and clinicopathological features.

both within the tumour mass (ILD) and in the peritumoural area (PLD) [5,14-17]. Higher ILD correlated with a more aggressive tumour phenotype and high LVD was predictor of poor survival $(\mathrm{p}=0.045)$. However, when laryngeal and pharyngeal tumours were considered separately, these associations were observed only in laryngeal carcinomas (LVD and survival, $\mathrm{p}=0.027$ ), suggesting a different contribution of lymphangiogenesis at different anatomic sites. While heterogeneous results can be found in the literature regarding this question, $[5,14-17]$ our data leave evidence of a more relevant role of lymphangiogenesis in the laryngeal carcinoma subgroup. Anatomic characteristics could account for these differences. The pharynx is a lymph vessel rich area so that virtually all tumours arising there might benefit from this circumstance. In our series $96 \%$ pharyngeal tumours were LVD positive and $90 \%$ ILD positive. Thus, no differences can be expected to be observed regarding lymph vessel density impact on outcome. In contrast, the larynx (specially the glottis) is a lymph vessel poor region and those tumours able to induce lymphangiogenesis ( $57 \%$ ILD positive cases in our series) might show a selective advantage regarding spread and thus a poorer outcome than those that remain vessel free.

In reference to the existence of dividing cells among lymphatic endothelial cells, we found evidence of at least one proliferative endothelial lymphatic cell in some tumour sections, although their proportion was low. These results are in line with previous reports that point to the existence of an active lymphangiogenesis process in HNSCC $[5,15]$.

The correlation between ILD and nodal involvement supports the role of intratumoural lymph vessels as a possible route for the spread of tumours to local lymph nodes. Interestingly, tumour emboli (LI, lymphatic invasion) were observed within lymphatics in $43 \%$ of tumour samples, a higher frequency than previously reported $[5,15,17,18]$. Importantly, LI correlated with tumour size, nodal involvement, tumour stage and a reduced survival rate. 


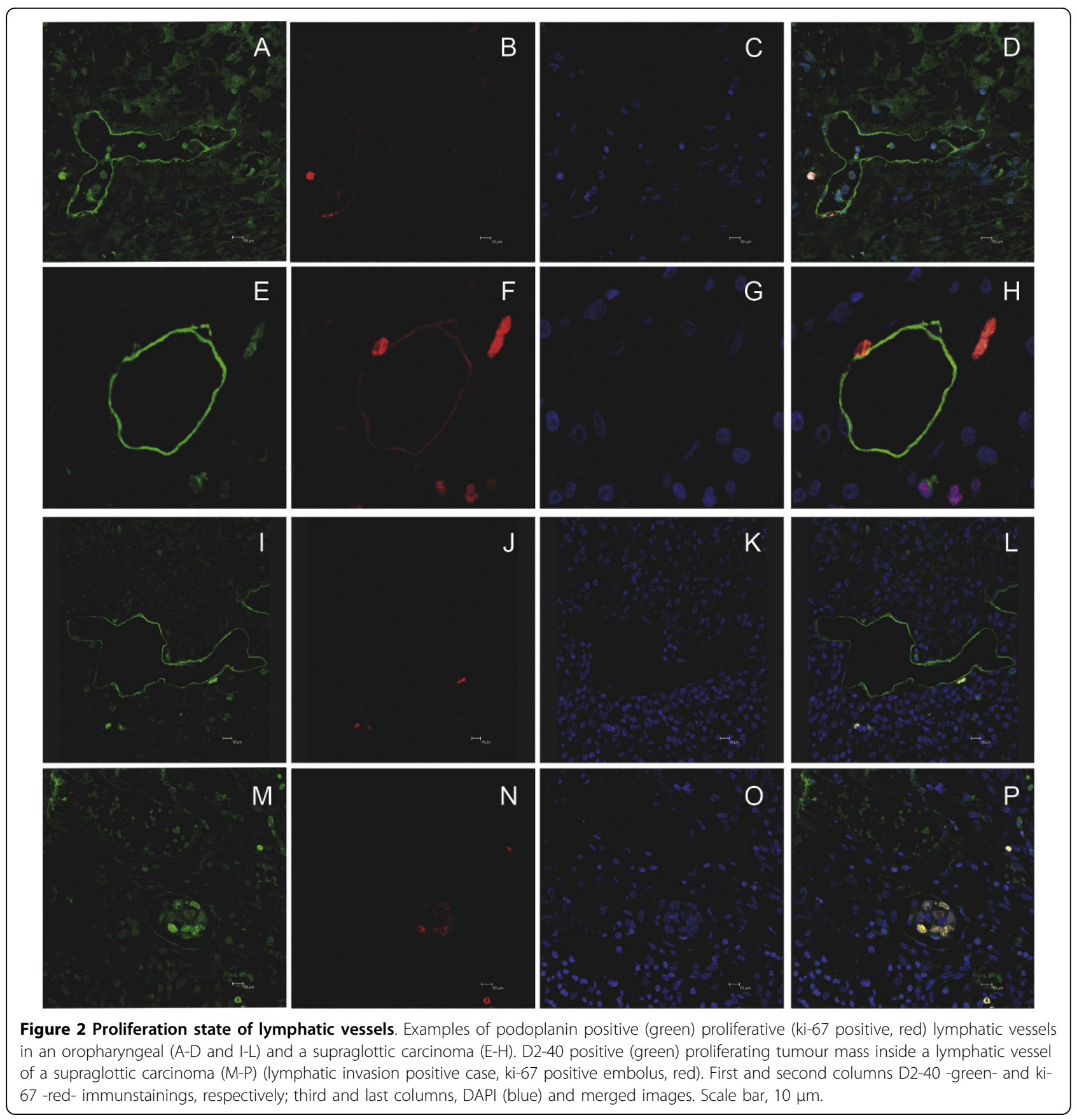

Table 5 Impact of the lymphatic vessel density on survival

\begin{tabular}{lcccccc}
\hline & \multicolumn{2}{c}{ ALL CASES $(\mathbf{N}=\mathbf{1 0 4})$} & \multicolumn{2}{c}{ Larynx $(\mathbf{N}=\mathbf{5 4})$} & \multicolumn{2}{c}{ Pharynx $(\mathbf{N}=\mathbf{5 0})$} \\
\cline { 2 - 7 } & Median & $\mathbf{p}$ & Median & $\mathbf{p}$ & Median & $\mathbf{p}$ \\
\hline ILD & 13.22 & 0.15 & 7.56 & 0.3262 & 18.42 & 0.6831 \\
PLD & 22.67 & 0.3898 & 20.78 & 0.6321 & 26.92 & 0.7139 \\
LVD & 22.04 & $\mathbf{0 . 0 4 5}$ & 20.78 & $\mathbf{0 . 0 2 7}$ & 22.98 & 0.334 \\
LI & & $\mathbf{0 . 0 4 5}$ & & $\mathbf{0 . 0 1 5}$ & & 0.5933
\end{tabular}

Kaplan Meier disease specific survival analysis p-values for ILD, PLD, LVD dichotomized according to their median values - in the whole population, laryngeal and pharyngeal tumours
Lymphatic cell proliferation and tumour emboli formation may be rare events, difficult to detect by examining small sections of archival tissue at a single time point. Despite this, the detection of proliferating lymphatics in carcinoma tissue and of tumour emboli within lymphatics constitutes considerable evidence for the presence of active lymphangiogenesis in this type of malignancy. Additionally, we present evidence of a true lymphangiogenic process in the larynx, since lymphatics were detected in pT1 tumours arising in the glottis, a 


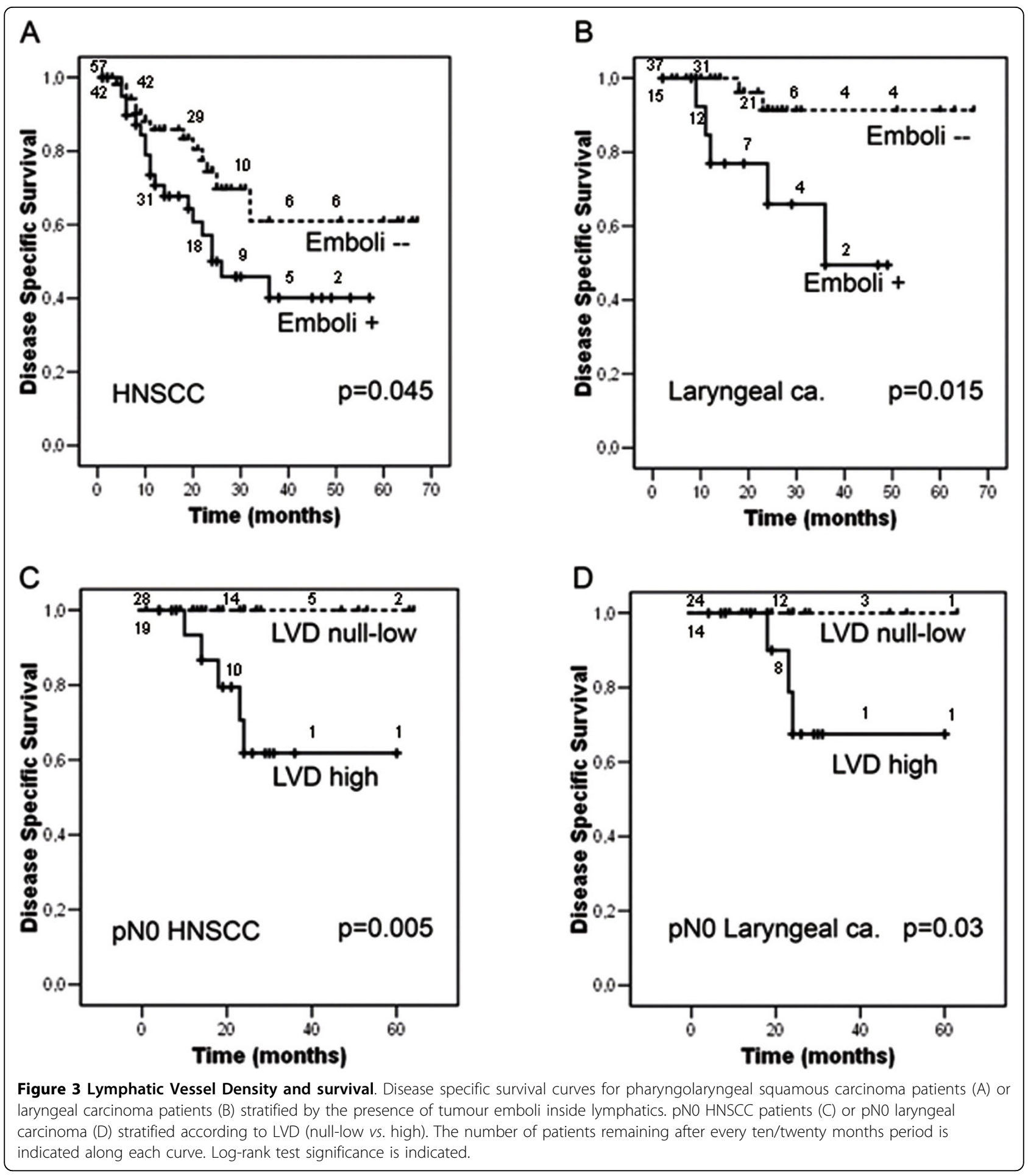

physiologically lymph vessel free site. This observation would indicate the formation of new vessels in these cases. On the other hand, the presence of lymphatics in the pT2-4 glottic tumours would not necessarily point to this phenomenon, since vessels might also result from their being entrapped as growing tumours reached the vessel rich areas, such as the paraglottic space, supraglottis or subglottis.

Controversial results can be found in the literature regarding the specific contribution of intra and peritumoural lymphatic vessels to tumour spread. Functional studies in animal models have shown that 
intratumoural lymphatic vessels are compressed and nonfunctional. However, some studies on human tumour samples reveal that intratumoural lymphatics are involved in nodal metastasis and that ILD is a significant indicator of poor prognosis $[5,14,17]$. Other authors describe an association of high PLD with shorter disease-free survival,[16,19] whereas a correlation between higher PLD and a more favourable outcome has also been reported[17]. These heterogeneous results could arise from the different methods used to quantify LVD and could reflect subjective factors and the intrinsic difficulties found in intra $v s$. peritumoural vessel identification when studying single tissue sections taken at a single time point, which might yield a misleading vessel classification, limiting the potential value of these variables. Although both ILD and PLD contribute to tumour spread to lymph nodes, from our experience, LVD counting (regardless of their location with respect to the tumour) is more reproducible and might be a more reliable marker of poor prognosis, especially within the laryngeal subgroup.

Remarkably, although nodal involvement had a negative prognostic value, this prediction could be refined among the pNO laryngeal tumour population, according to their LVD. Thus, significant differences on survival times were found for cases showing a high LVD (poorer outcome). This observation was also made in the whole series of carcinomas but not in the pharyngeal subgroup, and might be a useful tool in the pNO laryngeal cancer patients' clinical management.

\section{Conclusions}

In summary, our results suggest a differential implication of lymphangiogenesis in tumours arising from different anatomical head and neck sites. We propose that LVD assessment and tumour emboli detection using the D2-40 antibody should be considered as potential prognostic tools for laryngeal tumours. This would merit further large-scale validation analyses for the eventual future incorporation of these potential markers into clinical practice.

\section{Acknowledgements}

We thank Olivia García Suarez and Marta Sánchez Pitiot for their technical help and Dr Gloria H Su for critical reading of the manuscript. This study was supported by grant from Programa Ramón y Cajal -Ministerio de Educación y Ciencia, Spain (MVG), FICYT (PC 04-20) (CS) and Obra Social CajAstur (DGC). The Instituto Universitario de Oncología is supported by Obra Social Cajastur.

\section{Author details}

${ }^{1}$ Instituto Universitario de Oncología del Principado de Asturias (IUOPA), Universidad de Oviedo, Asturias, Spain. ${ }^{2}$ Servicio de Otorrinolaringología, Hospital Universitario Central de Asturias (HUCA), Oviedo (Asturias), Spain.
${ }^{3}$ Servicio Anatomía Patológica, Hospital Universitario Central de Asturias (HUCA), Oviedo (Asturias), Spain.

\section{Authors' contributions}

DGC carried out the IHC studies and vessel counting and helped to draft the manuscript. JPR participated in its design and contributed to clinical data collection and results interpretation. AA contributed to the pathological assessment of tissue sections. CSN participated in its design and coordination. MVG conceived the study, coordinated and supervised its progression, carried out vessel counting, performed the statistical analysis and wrote the manuscript. All authors read and approved the final manuscript.

\section{Competing interests}

The authors declare that they have no competing interests.

Received: 10 November 2009 Accepted: 10 August 2010

Published: 10 August 2010

\section{References}

1. Stewart B, Kleihues P: World Cancer Report Geneva: International Agency for Research on Cancer 2003.

2. Wicki A, Christofori G: The potential role of podoplanin in tumour invasion. British journal of cancer 2007, 96(1):1-5.

3. Clarijs R, Ruiter DJ, de Waal RM: Lymphangiogenesis in malignant tumours: Does it occur? The Journal of pathology 2001, 193(2):143-146.

4. Williams CS, Leek RD, Robson AM, Banerji S, Prevo R, Harris AL, Jackson DG: Absence of lymphangiogenesis and intratumoural lymph vessels in human metastatic breast cancer. The Journal of pathology 2003, 200(2):195-206.

5. Beasley NJ, Prevo R, Banerji S, Leek RD, Moore J, van Trappen P, Cox G, Harris AL, Jackson DG: Intratumoral lymphangiogenesis and lymph node metastasis in head and neck cancer. Cancer research 2002, 62(5):1315-1320

6. Pepper MS: Lymphangiogenesis and tumor metastasis: myth or reality? Clin Cancer Res 2001, 7(3):462-468.

7. Leu AJ, Berk DA, Lymboussaki A, Alitalo K, Jain RK: Absence of functional lymphatics within a murine sarcoma: a molecular and functional evaluation. Cancer research 2000, 60(16):4324-4327.

8. Stacker SA, Caesar C, Baldwin ME, Thornton GE, Williams RA, Prevo R, Jackson DG, Nishikawa S, Kubo H, Achen MG: VEGF-D promotes the metastatic spread of tumor cells via the lymphatics. Nature medicine 2001, 7(2):186-191.

9. Evangelou E, Kyzas PA, Trikalinos TA: Comparison of the diagnostic accuracy of lymphatic endothelium markers: Bayesian approach. Mod Pathol 2005, 18(11):1490-1497.

10. Van der Auwera I, Cao Y, Tille JC, Pepper MS, Jackson DG, Fox SB, Harris AL, Dirix LY, Vermeulen PB: First international consensus on the methodology of lymphangiogenesis quantification in solid human tumours. British journal of cancer 2006, 95(12):1611-1625.

11. Kahn HJ, Marks A: A new monoclonal antibody, D2-40. for detection of lymphatic invasion in primary tumors. Laboratory investigation; a journal of technical methods and pathology 2002, 82(9):1255-1257.

12. McShane LM, Altman DG, Sauerbrei W, Taube SE, Gion M, Clark GM: Reporting recommendations for tumor marker prognostic studies (REMARK). Journal of the National Cancer Institute 2005, 97(16):1180-1184.

13. Yuan P, Temam S, El-Naggar A, Zhou X, Liu DD, Lee JJ, Mao L: Overexpression of podoplanin in oral cancer and its association with poor clinical outcome. Cancer 2006, 107(3):563-569.

14. Audet N, Beasley NJ, MacMillan C, Jackson DG, Gullane PJ, Kamel-Reid S: Lymphatic vessel density, nodal metastases, and prognosis in patients with head and neck cancer. Archives of otolaryngology-head \& neck surgery 2005, 131(12):1065-1070.

15. Kyzas PA, Geleff S, Batistatou A, Agnantis NJ, Stefanou D: Evidence for lymphangiogenesis and its prognostic implications in head and neck squamous cell carcinoma. The Journal of pathology 2005, 206(2):170-177.

16. Franchi A, Gallo O, Massi D, Baroni G, Santucci M: Tumor lymphangiogenesis in head and neck squamous cell carcinoma: a morphometric study with clinical correlations. Cancer 2004 101(5):973-978. 
17. Maula SM, Luukkaa M, Grenman R, Jackson D, Jalkanen S, Ristamaki R: Intratumoral lymphatics are essential for the metastatic spread and prognosis in squamous cell carcinomas of the head and neck region. Cancer research 2003, 63(8):1920-1926.

18. Kyzas PA, Stefanou D, Batistatou A, Agnantis NJ, Nakanishi Y, Hirohashi S, Charalabopoulos $K$ : Dysadherin expression in head and neck squamous cell carcinoma: association with lymphangiogenesis and prognostic significance. The American journal of surgical pathology 2006, 30(2):185-193.

19. Longatto Filho A, Oliveira TG, Pinheiro C, de Carvalho MB, Curioni OA, Mercante AM, Schmitt FC, Gattas GJ: How useful is the assessment of lymphatic vascular density in oral carcinoma prognosis? World I Surg Oncol 2007, 5:140

20. Sobin LH, Wittekind C: TNM classification of malignant tumors New York: Wiley-Liss, 62002.

Pre-publication history

The pre-publication history for this paper can be accessed here: http://www.biomedcentral.com/1471-2407/10/416/prepub

doi:10.1186/1471-2407-10-416

Cite this article as: Garcia-Carracedo et al.: Prognostic significance of lymphangiogenesis in pharyngolaryngeal carcinoma patients. BMC Cancer 2010 10:416.

\section{Submit your next manuscript to BioMed Central} and take full advantage of:

- Convenient online submission

- Thorough peer review

- No space constraints or color figure charges

- Immediate publication on acceptance

- Inclusion in PubMed, CAS, Scopus and Google Scholar

- Research which is freely available for redistribution

Submit your manuscript at www.biomedcentral.com/submit 